\title{
DIVERSIDADE DE NYMPHALIDAE NA ESTAÇÃO ECOLÓGICA DE IQUÊ, MUNICÍPIO DE JUÍNA, ESTADO DE MATTO GROSSO, BRASIL
}

\author{
Cibele Madalena Xavier Ribeiro ${ }^{1}$, Alberto Dorval $^{2}$, Otávio Peres Filho ${ }^{2}$, Reginaldo Brito da \\ Costa $^{2}$
}

\footnotetext{
${ }^{1}$ Programa de Pós-Graduação em Ciências Florestais e Ambientais, FENF, Universidade Federal de Mato Grosso - UFMT

${ }^{2}$ Departamento de Engenharia Florestal, Universidade Federal de Mato Grosso, UFMT, e-mail: a.dorval@ hotmail.com
}

\section{RESUMO}

O presente estudo objetivou analisar a fauna de borboletas frugívoras (Nymphalidae: Lepidoptera) na Estação Ecológica de Iquê, no município de Juína, estado de Mato Grosso, através de um levantamento não-perturbatório visando contribuir para o conhecimento qualitativo e quantitativo das espécies ocorrentes na unidade de conservação. Foram utilizadas 30 armadilhas do tipo Van Someren-Rydon, distribuídas em três unidades amostrais. Observou-se uma diversidade de 75 espécies, distribuídas em seis subfamílias de Nymphalidae com 605 espécimes coletados. Catonephele acontius, Temenis laothe e Eunica sp. 1 (Biblidinae), Archaeoprepona demophon (Charaxinae), Fosterinaria sp., Harjesia obscura, Hermeuptychia hermes, Pseudodebis valentina e Taygetis virgilia (Satyrinae) foram as espécies mais abundantes em número de indivíduos amostrados. $\mathrm{Na}$ análise faunística ocorreram 13 espécies dominantes, constantes, muito freqüentes e muito abundantes. $\mathrm{O}$ índice de diversidade é considerado alto para a região e a equitatividade de 0,80 mostrou uma área bem preservada onde as espécies de borboletas encontram condições ambientais favoráveis para sua distribuição, manutenção e reprodução.

Palavras-chave: Lepidoptera, áreas protegidas, armadilhas Van Someren-Rydon

\section{DIVERSITY OF NYMPHALIDAE IN IQUÊ ECOLOGICAL STATION, JUÍNA MUNICIPALITY, MATO GROSSO STATE, BRAZIL}

\begin{abstract}
This study aimed to analyze the fauna of fruit-feeding butterflies (Nymphalidae: Lepidoptera) in the Ecological Station Iquê, Juína municipality, Mato Grosso state, Brazil, through a non-disturbing survey to contribute to the knowledge of qualitative and quantitative species in the protected area. Thirty Van Someren-Rydon traps were used, divided into three sampling units. There was a diversity of 75 species in six subfamilies of Nymphalidae with 605 specimens. Catonephele acontius, Temenis laothe and Eunica sp. 1 (Biblidinae), Archaeoprepona demophon (Charaxinae), Fosterinaria sp., Harjesia obscure, Hermeuptychia hermes, Pseudodebis valentina and Taygetis virgilia (Satyrinae) were the most abundant species in number of individuals sampled. In the faunistic analysis, there were 13 dominant, constant, very frequent and abundant species. Diversity index is considered high for the region and the evenness of 0.80 shows a well-preserved area where species of butterflies have favorable environmental conditions for their distribution, maintenance and reproduction.
\end{abstract}

Key words: Lepidoptera, protected areas, Van Someren-Rydon traps 


\section{INTRODUÇÃO}

As comunidades tropicais são caracterizadas pela alta riqueza de espécies e pelo importante papel que desempenham no desenvolvimento biológico evolutivo (DeVries \& Walla, 2001). Devido à destruição dos hábitats, os estudos sobre a diversidade de espécies têm se tornado de vital importância para a compreensão das comunidades biológicas e sua conservação (Purvis \& Hector, 2000), pois o conhecimento sobre o funcionamento dos ricos e complexos sistemas tropicais é incipiente devido aos poucos estudos abordando a distribuição das abundâncias de espécies no espaço e tempo (MacArthur, 1972; Elton, 1973). De acordo com DeVries e Walla (2001), os trabalhos mais recentes em regiões tropicais têm se concentrado em desenvolver técnicas que estimem a riqueza de espécies, levantamentos rápidos de riqueza de espécies em diferentes hábitats ou modelagem da dinâmica das comunidades.

Os lepidópteros, uma das principais ordens de insetos quanto à riqueza de espécies, são importantes para estudos ambientais sobre a biodiversidade (Landau et al., 1999). Entre os membros dessa Ordem, as espécies de borboletas da família Nymphalidae destacam-se pela diversidade de hábitos alimentares, que incluem frutas fermentadas, excrementos, exsudatos de plantas e animais em decomposição e, também, pelos aspectos morfológicos, sendo facilmente amostrados e reconhecíveis em campo (DeVries, 1987; Freitas et al., 2003).

Estas características fazem com que essas espécies sejam facilmente coletadas, o que possibilita a amostragem de duas ou mais áreas simultaneamente e, por isso, são muito úteis na elaboração de inventários locais não destrutivos, adequando-se satisfatoriamente aos programas de monitoramento ambiental (Brown Jr., 1991, 1997; Ribeiro, 2006). No Brasil, borboletas e formigas tornaram-se, especialmente úteis, para estudos de monitoramento ambiental (Brown Jr. \& Freitas, 2000a; Shoereder et al., 2004) por serem muito diversificadas, facilmente amostradas, identificadas e abundantes o ano inteiro, especialistas em recursos específicos, por possuírem fidelidade ecológica e por apresentarem respostas rápidas às alterações ambientais (Freitas et al., 2003). Apesar da grande diversidade de espécies de borboletas que ocorrem em ambientes naturais, pouco se conhece sobre sua distribuição geográfica e bioecologia nas diferentes regiões do país. Santos et al. (2008) afirmaram que dos 134 inventários de borboletas publicados no Brasil, 30 foram inventários regionais e 48 locais e que apenas $22 \%$ foram realizados dentro de unidades de conservação.

No estado de Mato Grosso, o conhecimento sobre a ocorrência e a distribuição da entomofauna regional é bastante incipiente para muitos grupos, entre estes as borboletas. A utilização de listas regionais de espécies é uma ferramenta prática no auxílio de estudos de diversidade taxonômica, genética e ecológica de um local ou região, disponibilizando informações que subsidiarão programas de conservação da biodiversidade.

Nesse sentido, o presente estudo objetivou analisar a diversidade e a composição da entomofauna de ninfalídeos na estação ecológica de Iquê, localizada na Amazônia Matogrossense através de levantamentos não perturbatórios de modo a contribuir para o conhecimento qualitativo e quantitativo das espécies desta família de lepidópteros.

\section{MATERIAL E METÓDOS}

O estudo foi conduzido na Estação Ecológica de Iquê, com uma área 200 mil hectares, localizada nas coordenadas geográficas $11^{\circ} 30^{\prime}-12^{\circ} 15^{\prime} \mathrm{S}$ e $58^{\circ} 40^{\prime}$ $59^{\circ} 20^{\prime} \mathrm{W}$, no município de Juína, noroeste do estado do Mato Grosso. O clima da região é 
tropical quente e úmido (Am) com temperaturas médias anuais superiores a 28 ${ }^{\circ} \mathrm{C}$. A unidade de conservação possui um relevo plano a suavemente ondulado, com uma rede de drenagem diversificada. As amostragens foram conduzidas junto à sede da estação, às margens da MT-319/BR-174, estabelecendo-se transectos de 1.000 metros e instalaram-se dez armadilhas portáteis do tipo Van Someren-Rydon (Modelo adaptado de DeVries, 1987) por transecto, perfazendo um total de 30 armadilhas. Para minimizar a probabilidade de influência sobre a coleta das borboletas, as armadilhas foram dispostas linearmente aos pares, a uma distância média de 100 metros entre si, sendo cinco instaladas do lado direito e cinco do lado esquerdo do transecto, de acordo com o proposto por DeVries \& Walla (2001).

As coletas foram realizadas entre os meses de janeiro e julho de 2008, com três coletas em cada mês, para verificar os padrões de sazonalidade das borboletas. Em cada mês as armadilhas foram mantidas iscadas por um período de seis dias, perfazendo um total de 360 horas, ou 10.800 horas/armadilha, considerando-se 10 horas de efetiva amostragem/dia. As armadilhas foram iscadas com uma mistura padronizada de banana amassada (cerca de três $\mathrm{kg}$ por preparo) e caldo de cana (1 litro) e a preparação das iscas ocorreu sempre 48 horas antes do início das coletas. Em campo, as iscas foram acondicionadas em potes plásticos com as tampas perfuradas e trocadas a cada 48 horas, para que se mantivessem atrativas.

Os espécimes coletados, antes de serem liberados, foram marcados na face ventral das asas anteriores com uma caneta não-tóxica para que as possíveis recoletas fossem contabilizadas e todos os indivíduos fotografados, formando assim, um banco de imagens dos indivíduos observados, coletados e recoletados. Para as análises qualitativas, quantitativas e nos cálculos dos índices faunísticos utilizou-se o software ANAFAU (Moraes \& Silveira Neto, 2002). $O$ índice de diversidade foi calculado através do programa Stastical Ecology (Ludwig \& Reynolds, 1988). As médias de coletas dos indivíduos das espécies mais representativas foram submetidas ao teste de Tukey a 5\% de probabilidade. A identificação taxonômica dos espécimes coletados foi efetuada mediante comparação com os exemplares da coleção entomológica do Instituto de Biologia (IB), da Universidade Federal de Mato Grosso.

\section{RESULTADOS E DISCUSSÃO}

No estudo contabilizaram-se 605 indivíduos coletados, distribuídos em 38 gêneros, 75 espécies e seis subfamílias de Nymphalidae. Satyrinae com 16 (42,11\%) gêneros, $40 \quad(53,33 \%)$ espécies e 288 $(47,60 \%)$ indivíduos amostrados constituiuse na subfamília mais representativa, enquanto Heliconiinae foi a menos representativa entre as subfamílias em quantidade de gênero, espécie e de indivíduos (Tabela 1). Em função deste levantamento ser o primeiro abrangendo aspectos qualitativos e quantitativos com borboletas realizado na região, não foi possível levantar as causas que possam ter influenciado na maior ou menor quantidade de alguns gêneros ou espécies de borboletas frugívoras nas armadilhas.

Os resultados observados são condizentes com as afirmações de Ribeiro (2006) de que ambientes de ecótonos podem abrigar maior diversidade de espécies de satiríneos e as áreas de capoeira e estágios iniciais de regeneração que apresentam uma grande diversidade de espécies de plantas hospedeiras são preferidas por indivíduos da subfamília Biblidinae. Segundo Downes (1973), os ninfalídeos mostram forte resposta aos odores e os insetos se manifestam individualmente ou mostram agregações em direção aos sítios onde há 
oferta de recursos. Nesse contexto, a ausência dos indivíduos de várias espécies pode estar relacionada à oferta mais atrativa de outras fontes de recursos alimentares, pois borboletas adultas tendem a ser menos específicas e mais oportunistas que suas formas imaturas no uso dos substratos alimentares (Gilbert \& Singer, 1975) e as atividades de procura e coleta dos itens alimentares entre as espécies, entre os sexos notadamente pela função reprodutiva, são afetadas pelas condições ambientais, onde naturalmente há busca pelo equilíbrio entre esforço e recompensa alcançada por determinada estratégia (Vieira, 2004).

Durante o período de amostragem foram coletadas, registradas e identificadas 76 espécies pertencentes a seis subfamílias de Nymphalidae, totalizando 605 indivíduos. Entre as espécies registradas, $17(22,66 \%)$ ocorreram com densidades populacionais mais expressivas e representaram $67,10 \%$ dos indivíduos coletados, enquanto 58 $(77,34 \%)$ ocorreram em populações com menos de dez indivíduos e representaram $32,90 \%$ do total de indivíduos amostrados.
As espécies Catonephele acontius (19,67\%), Fosterinaria sp. (7,93\%), Harjesia sp. (4,30\%), Archaeoprepona demophon (3,97\%), Temenis laothe (3,80\%), Harjesia obscura (3,64\%), Taygetis tripuctata (3,31\%), Taygetis virgilia (3,14\%), Hermeuptychia hermes (2,98\%), Catonephele numilia (2,81\%), Morpho achilles (2,48\%) foram, quantitativamente, as mais representativas em indivíduos amostrados (Tabela 2). A maior abundância relativa dos satiríneos, principalmente, nos meses com altas temperaturas, quando comparado com as demais subfamílias, é explicada pelo comportamento das espécies dessa subfamília, já que o aumento da temperatura aumenta também a atividade dos indivíduos, que tendem a voar mais próximos ao solo (DeVries, 1987). Segundo Brown Jr. \& Freitas (2000a), os satiríneos, tão presentes neste levantamento, relacionam-se negativamente com a perturbação antrópica, indicando o grau de integridade da paisagem na Estação Ecológica de Iquê.

Tabela 1. Gênero, espécie e quantidade de indivíduos de subfamílias de Nymphalidae coletados com armadilhas portáteis do tipo Van Someren-Rydon instaladas na Estação Ecológica de Iquê, Juína-MT, 2008.

\begin{tabular}{l|c|c|c|c|c|c}
\hline Subfamílias & Gêneros & $\%$ & Espécies & $\%$ & Indivíduos & $\%$ \\
\hline Biblidinae & 9 & 23,68 & 16 & 21,33 & 219 & 36,20 \\
Charaxinae & 5 & 13,16 & 9 & 12,00 & 42 & 6,94 \\
Heliconiinae & 1 & 2,63 & 1 & 1,33 & 1 & 0,17 \\
Morphinae & 6 & 15,79 & 8 & 10,67 & 38 & 6,28 \\
Nymphalinae & 1 & 2,63 & 1 & 1,33 & 6 & 0,99 \\
Satyrinae & 16 & 42,11 & 40 & 53,33 & 288 & 47,60 \\
Não identificadas & - & - & - & - & 11 & 1,82 \\
\hline Total & 38 & 100 & 75 & 100 & 605 & 100 \\
\hline
\end{tabular}


Tabela 2. Subfamília, gênero, espécie e quantidade de indivíduos coletados na Estação Ecológica de Iquê, Juína-MT, 2008.

\begin{tabular}{|c|c|c|c|c|c|c|}
\hline Subfamília/Espécies & I & $\mathrm{F}(\%)$ & $\mathrm{D}$ & $\mathrm{D}^{*}$ & $\mathrm{C}$ & A \\
\hline \multicolumn{7}{|l|}{ BIBLIDINAE } \\
\hline Agrias pericles (Bates, 1860) & 1 & $0,17(\mathrm{pf})$ & nd & nd & $\mathrm{z}$ & $\mathrm{r}$ \\
\hline Callicore sp. & 1 & $0,17(\mathrm{pf})$ & nd & nd & $\mathrm{z}$ & $\mathrm{r}$ \\
\hline Callicore astarte (Cramer, 1779) & 1 & $0,17(\mathrm{pf})$ & nd & nd & $\mathrm{z}$ & $\mathrm{r}$ \\
\hline Catonephele acontius (Linnaeus, 1771) & 119 & $19,67(\mathrm{mf})$ & $\mathrm{d}$ & $\mathrm{d}$ & $\mathrm{w}$ & ma \\
\hline Catonephele numilia (Cramer, 1776) & 17 & $2,81(\mathrm{mf})$ & $\mathrm{d}$ & $\mathrm{d}$ & $\mathrm{w}$ & ma \\
\hline Ectima iona (Hewitson, 1848) & 3 & $0,50(\mathrm{pf})$ & nd & nd & $\mathrm{z}$ & $\mathrm{r}$ \\
\hline Eunica sp. 1 & 21 & $3,47(\mathrm{mf})$ & $\mathrm{d}$ & $\mathrm{d}$ & $\mathrm{W}$ & ma \\
\hline Eunica sp. 2 & 2 & $0,33(\mathrm{pf})$ & nd & nd & $\mathrm{Z}$ & $\mathrm{r}$ \\
\hline Eunica bechina (Hewitson, 1852) & 1 & $0,17(\mathrm{pf})$ & nd & nd & $\mathrm{z}$ & $\mathrm{r}$ \\
\hline Eunica migdonya (Godart, 1824) & 1 & $0,17(\mathrm{pf})$ & nd & nd & $\mathrm{z}$ & $\mathrm{r}$ \\
\hline Eunica phasis (Felder \& R. Felder, 1862) & 1 & $0,17(\mathrm{pf})$ & nd & nd & $\mathrm{z}$ & $\mathrm{r}$ \\
\hline Eunica verônica (Bates, 1864) & 1 & $0,17(\mathrm{pf})$ & nd & nd & $\mathrm{z}$ & $\mathrm{r}$ \\
\hline Nessaea obrinus (Linnaeus, 1758) & 12 & $1,98(\mathrm{mf})$ & $\mathrm{d}$ & $\mathrm{d}$ & $\mathrm{y}$ & $\mathrm{a}$ \\
\hline Nica flavilla (Godart, 1824) & 11 & $1,82(\mathrm{mf})$ & $\mathrm{d}$ & $\mathrm{d}$ & $\mathrm{y}$ & $\mathrm{a}$ \\
\hline Temenis laothe (Cramer, 1777) & 23 & $3,80(\mathrm{mf})$ & $\mathrm{d}$ & $\mathrm{d}$ & $\mathrm{W}$ & ma \\
\hline Pyrrhogyra amphiro (Bates, 1865) & 4 & $0,66(\mathrm{pf})$ & nd & nd & $\mathrm{z}$ & $\mathrm{d}$ \\
\hline \multicolumn{7}{|l|}{ CHARAXINAE } \\
\hline Archaeoprepona demophon (Linnaeus, 1758) & 24 & $3,97(\mathrm{mf})$ & $\mathrm{d}$ & $\mathrm{d}$ & $\mathrm{w}$ & $\mathrm{ma}$ \\
\hline Archaeoprepona amphimacus (Fabricius, 1775) & 1 & $0,17(\mathrm{pf})$ & nd & nd & $\mathrm{z}$ & $\mathrm{r}$ \\
\hline Fontainea ryphea (Cramer, 1775) & 2 & $0,33(\mathrm{pf})$ & nd & nd & $\mathrm{z}$ & $\mathrm{r}$ \\
\hline Hypna clytmnestra (Cramer, 1777) & 1 & $0,17(\mathrm{pf})$ & nd & nd & $\mathrm{z}$ & $\mathrm{r}$ \\
\hline Memphis sp. 1 & 3 & $0,50(\mathrm{pf})$ & nd & nd & $\mathrm{z}$ & $\mathrm{r}$ \\
\hline Memphis moruus (Fabricius, 1775) & 4 & $0,66(\mathrm{pf})$ & nd & nd & $\mathrm{z}$ & $\mathrm{d}$ \\
\hline Memphis philumena (Doubleday, 1849) & 1 & $0,17(\mathrm{pf})$ & nd & nd & $\mathrm{z}$ & $\mathrm{r}$ \\
\hline Zaretis isidora (Cramer, 1779) & 3 & $0,50(\mathrm{pf})$ & nd & nd & $\mathrm{z}$ & $\mathrm{d}$ \\
\hline \multicolumn{7}{|l|}{ CHARAXINAE } \\
\hline Zaretis itys (Westwood, 1850) & 3 & $0,50(\mathrm{pf})$ & nd & nd & $\mathrm{z}$ & $\mathrm{r}$ \\
\hline \multicolumn{7}{|l|}{ HELICONIINAE } \\
\hline Heliconius sp. & 1 & $0,17(\mathrm{pf})$ & nd & nd & $\mathrm{z}$ & $\mathrm{r}$ \\
\hline \multicolumn{7}{|l|}{ MORPHOINAE } \\
\hline Anthirrea taygetina (Butler, 1868) & 1 & $0,17(\mathrm{pf})$ & nd & nd & $\mathrm{z}$ & $\mathrm{r}$ \\
\hline Bia actorion (Linnaeus, 1763) & 4 & $0,66(\mathrm{pf})$ & nd & nd & $\mathrm{z}$ & $\mathrm{d}$ \\
\hline \multicolumn{7}{|l|}{ MORPHOINAE } \\
\hline Caligo idomeneus (Linnaeus, 1758) & 2 & $0,33(\mathrm{pf})$ & nd & nd & $\mathrm{z}$ & $\mathrm{r}$ \\
\hline Caligo illioneus (Cramer, 1775) & 5 & $0,83(f)$ & nd & nd & $\mathrm{z}$ & $\mathrm{c}$ \\
\hline Caligopsis seleucida (Hewitson, (1877) & 1 & $0,17(\mathrm{pf})$ & nd & nd & $\mathrm{z}$ & $\mathrm{r}$ \\
\hline Morpho helenor (Cramer, 1776) & 8 & $1,32(\mathrm{f})$ & $\mathrm{d}$ & $\mathrm{d}$ & $\mathrm{y}$ & $\mathrm{c}$ \\
\hline Morpho achilles (Linnaeus, 1758) & 15 & $2,48(\mathrm{mf})$ & $\mathrm{d}$ & $\mathrm{d}$ & $\mathrm{W}$ & ma \\
\hline Opsiphanes invirae (Hübner, 1808) & 2 & $0,33(\mathrm{pf})$ & nd & nd & $\mathrm{z}$ & $\mathrm{r}$ \\
\hline \multicolumn{7}{|l|}{ NYMPHALINAE } \\
\hline Tigridia acesta (Linnaeus, 1758) & 6 & $0,99(f)$ & $\mathrm{d}$ & nd & $\mathrm{y}$ & $\mathrm{c}$ \\
\hline \multicolumn{7}{|l|}{ SATYRINAE } \\
\hline Caeruleuptychia cyanites (Butler, 1871) & 1 & $0,17(\mathrm{pf})$ & nd & nd & $\mathrm{z}$ & $\mathrm{r}$ \\
\hline Cissia confusa (Staudinger, 1867) & 2 & $0,33(\mathrm{pf})$ & nd & nd & $\mathrm{z}$ & $\mathrm{r}$ \\
\hline Cissia myncea (Cramer, 1780) & 2 & $0,33(\mathrm{pf})$ & nd & nd & $\mathrm{z}$ & $\mathrm{r}$ \\
\hline Cissia terrestris (Butler, 1867) & 5 & $0,83(\mathrm{f})$ & nd & nd & $\mathrm{y}$ & $\mathrm{c}$ \\
\hline
\end{tabular}


Tabela 2. Continuação....

\begin{tabular}{|c|c|c|c|c|c|c|}
\hline Subfamília/Espécies & $\mathrm{I}$ & $\mathrm{F}(\%)$ & $\mathrm{D}$ & $\mathrm{D}^{*}$ & $\mathrm{C}$ & A \\
\hline Cissia sp. 1 & 1 & $0,17(\mathrm{pf})$ & nd & nd & $\mathrm{z}$ & $\mathrm{r}$ \\
\hline Cissia sp. 2 & 1 & $0,17(\mathrm{pf})$ & nd & nd & $\mathrm{Z}$ & $\mathrm{r}$ \\
\hline Chloreuptchya arnaca (Fabricius, 1776) & 1 & $0,17(\mathrm{pf})$ & nd & nd & $\mathrm{z}$ & $\mathrm{r}$ \\
\hline Chloreuptychia herseis (Godart,1824) & 10 & $1,65(f)$ & $\mathrm{d}$ & $\mathrm{d}$ & $\mathrm{y}$ & $\mathrm{c}$ \\
\hline Erichthodes antonina & 1 & $0,17(\mathrm{pf})$ & nd & nd & $\mathrm{Z}$ & $\mathrm{r}$ \\
\hline Euptychia cleophes (Godman \& Salvin, 1889) & 2 & $0,33(\mathrm{pf})$ & nd & nd & $\mathrm{Z}$ & $\mathrm{r}$ \\
\hline Euptychia jesia (Butler, 1869) & 8 & $1,32(f)$ & $\mathrm{d}$ & $\mathrm{d}$ & $\mathrm{z}$ & $\mathrm{c}$ \\
\hline Euptychia mollina (Hübner, 1816) & 1 & $0,17(\mathrm{pf})$ & nd & nd & $\mathrm{z}$ & $\mathrm{r}$ \\
\hline Eupytchia westwoodii (Butler, 1866) & 2 & $0,33(\mathrm{pf})$ & nd & nd & $\mathrm{Z}$ & $\mathrm{r}$ \\
\hline Euptychia sp. 1 & 2 & $0,33(\mathrm{pf})$ & nd & nd & $\mathrm{z}$ & $\mathrm{r}$ \\
\hline Euptychiina sp. & 2 & $0,33(\mathrm{pf})$ & nd & nd & $\mathrm{z}$ & $\mathrm{r}$ \\
\hline Fosterinaria sp. & 48 & $7,93(\mathrm{mf})$ & $\mathrm{d}$ & $\mathrm{d}$ & $\mathrm{w}$ & ma \\
\hline Harjesia obscura (Butler, 1867) & 22 & $3,64(\mathrm{mf})$ & $\mathrm{d}$ & $\mathrm{d}$ & $\mathrm{w}$ & ma \\
\hline Harjesia sp. & 26 & $4,30(\mathrm{mf})$ & d & d & $\mathrm{w}$ & ma \\
\hline Hermeuptychia hermes (Fabricius, 1775) & 18 & $2,98(\mathrm{mf})$ & $\mathrm{d}$ & $\mathrm{d}$ & $\mathrm{w}$ & $\mathrm{ma}$ \\
\hline Magneuptychia sp. & 4 & $0,66(\mathrm{pf})$ & nd & $\mathrm{d}$ & $\mathrm{z}$ & $\mathrm{d}$ \\
\hline Megeoptychia antonoe (Cramer, 1775) & 2 & $0,33(\mathrm{pf})$ & nd & nd & $\mathrm{Z}$ & $\mathrm{r}$ \\
\hline Pareupthychia sp. & 1 & $0,17(\mathrm{pf})$ & nd & nd & $\mathrm{z}$ & $\mathrm{r}$ \\
\hline Pareuptychia ocirrhoe (Fabricius, 1776) & 5 & $0,83(f)$ & nd & nd & $\mathrm{Z}$ & $\mathrm{c}$ \\
\hline Parypthimoides sp. & 2 & $0,33(\mathrm{pf})$ & nd & nd & $\mathrm{z}$ & $\mathrm{r}$ \\
\hline Paryphthimoides phronius (Godart, 1824) & 2 & $0,33(\mathrm{pf})$ & nd & nd & $\mathrm{z}$ & $\mathrm{r}$ \\
\hline Pseudodebis valentina (Cramer, 1782) & 18 & $2,98(\mathrm{mf})$ & $\mathrm{d}$ & $\mathrm{d}$ & $\mathrm{w}$ & ma \\
\hline \multicolumn{7}{|l|}{ SATYRINAE } \\
\hline Postaygetis penelea (Cramer, 1777) & 6 & $0,99(f)$ & d & nd & $\mathrm{y}$ & $\mathrm{c}$ \\
\hline Splendeuptychia sp. & 6 & $0,99(f)$ & $\mathrm{d}$ & nd & $\mathrm{y}$ & $\mathrm{c}$ \\
\hline Taygetis Cleopatra (Felder \& Felder, 1867) & 2 & $0,33(\mathrm{pf})$ & nd & nd & $\mathrm{z}$ & $\mathrm{r}$ \\
\hline Taygetis laches (Fabricius, 1763) & 6 & $0,99(\mathrm{f})$ & $\mathrm{d}$ & nd & $\mathrm{z}$ & $\mathrm{c}$ \\
\hline Taygetis larua (Felder \& Felder, 1867) & 4 & $0,66(\mathrm{pf})$ & nd & nd & $\mathrm{Z}$ & d \\
\hline Taygetis mermeria (Cramer, 1776) & 5 & $0,83(f)$ & nd & nd & $\mathrm{z}$ & $\mathrm{c}$ \\
\hline Taygetis rectifascia (Weymer, 1907) & 4 & $0,66(\mathrm{pf})$ & nd & nd & $\mathrm{y}$ & $\mathrm{r}$ \\
\hline Taygetis sosis (Hopfer, 1874) & 4 & $0,66(\mathrm{pf})$ & nd & nd & $\mathrm{z}$ & d \\
\hline Taygetis thamyra (Cramer, 1779) & 12 & $1,98(\mathrm{mf})$ & $\mathrm{d}$ & $\mathrm{d}$ & $\mathrm{w}$ & $\mathrm{a}$ \\
\hline Taygetis tripunctata (Weymer, 1907) & 20 & $3,31(\mathrm{mf})$ & $\mathrm{d}$ & $\mathrm{d}$ & $\mathrm{w}$ & ma \\
\hline Taygetis virgilia (Cramer, 1776) & 19 & $3,14(\mathrm{mf})$ & $\mathrm{d}$ & $\mathrm{d}$ & $\mathrm{W}$ & ma \\
\hline Taygetis zippora (Butler, 1869) & 2 & $0,33(\mathrm{pf})$ & nd & nd & $\mathrm{z}$ & $\mathrm{r}$ \\
\hline Taygetis sp. & 4 & $0,66(\mathrm{pf})$ & nd & nd & $\mathrm{y}$ & $\mathrm{d}$ \\
\hline Ypthimoides sp. & 5 & $0,83(f)$ & nd & nd & $\mathrm{z}$ & $\mathrm{c}$ \\
\hline Não Identificadas & 11 & & & & & \\
\hline Total & 605 & 100 & & & & \\
\hline
\end{tabular}

(D) - Dominância (método Laroca \& Mielke); (D*) - Dominância (método Sakagami \& Laroca); (I) Indivíduos; (D) Dominante: dominante (d), não dominante (nd); Constância (C): constantes (w), acessórias (y), acidentais $(\mathrm{z})$; (F\%) Freqüência: muito freqüentes $(\mathrm{mf})$, freqüentes $(\mathrm{f})$, pouco freqüentes (pf); Abundância (A): muito abundantes (ma), abundantes (a), comuns (c), dispersas (d) e raras (r).

A análise faunística mostrou que Satyrinae e Biblidinae foram as subfamílias de Nymphalidae mais representativas em quantidade de espécies em relação aos principais índices faunísticos analisados (Tabela 3). 
Tabela 3. Quantidade de espécies das subfamílias de Nymphalidae dominantes, constantes, muito abundantes e muito freqüentes amostrados na Estação Ecológica de Iquê, Juína-MT, 2008 .

\begin{tabular}{l|c|c|c|c|c}
\hline \multirow{2}{*}{ Subfamílias } & \multicolumn{6}{c}{ Índices faunísticos } \\
\cline { 2 - 6 } & \multicolumn{2}{|c}{ Dominante } & Constante & Muito abundante & Muito frequente \\
\cline { 2 - 6 } & $\mathrm{d}$ & $\mathrm{d}^{1}$ & $\mathrm{w}$ & $\mathrm{ma}$ & $\mathrm{mf}$ \\
\hline Biblidinae & 6 & 6 & 4 & 4 & 6 \\
Charaxinae & 1 & 1 & 1 & 1 & 1 \\
Heliconiinae & 0 & 0 & 0 & 0 & 0 \\
Morphoinae & 2 & 2 & 1 & 1 & 1 \\
Nymphalinae & 1 & 0 & 0 & 0 & 0 \\
Satyrinae & 13 & 11 & 8 & 7 & 7 \\
\hline Total & 23 & 20 & 14 & 13 & 14 \\
\hline
\end{tabular}

Dominância: (d) - Método Laroca \& Mielke (1975); $\left(\mathrm{d}^{1}\right)$ - Sakagami \& Laroca (1971).

Entre as 75 espécies de borboletas analisadas pelo software ANAFAU, 52 $(69,33 \%)$ e $56(74,66 \%)$ espécies ocorreram de forma não dominante pelos métodos Laroca \& Mielke (1975) e Sakagami \& Laroca (1971), respectivamente. Em relação à constância, $51 \quad(67,10 \%)$ espécies ocorreram de forma acidental, $39(51,13 \%)$ com ocorrência rara e $47(61,84 \%)$ foram pouco freqüientes (Figura 1).

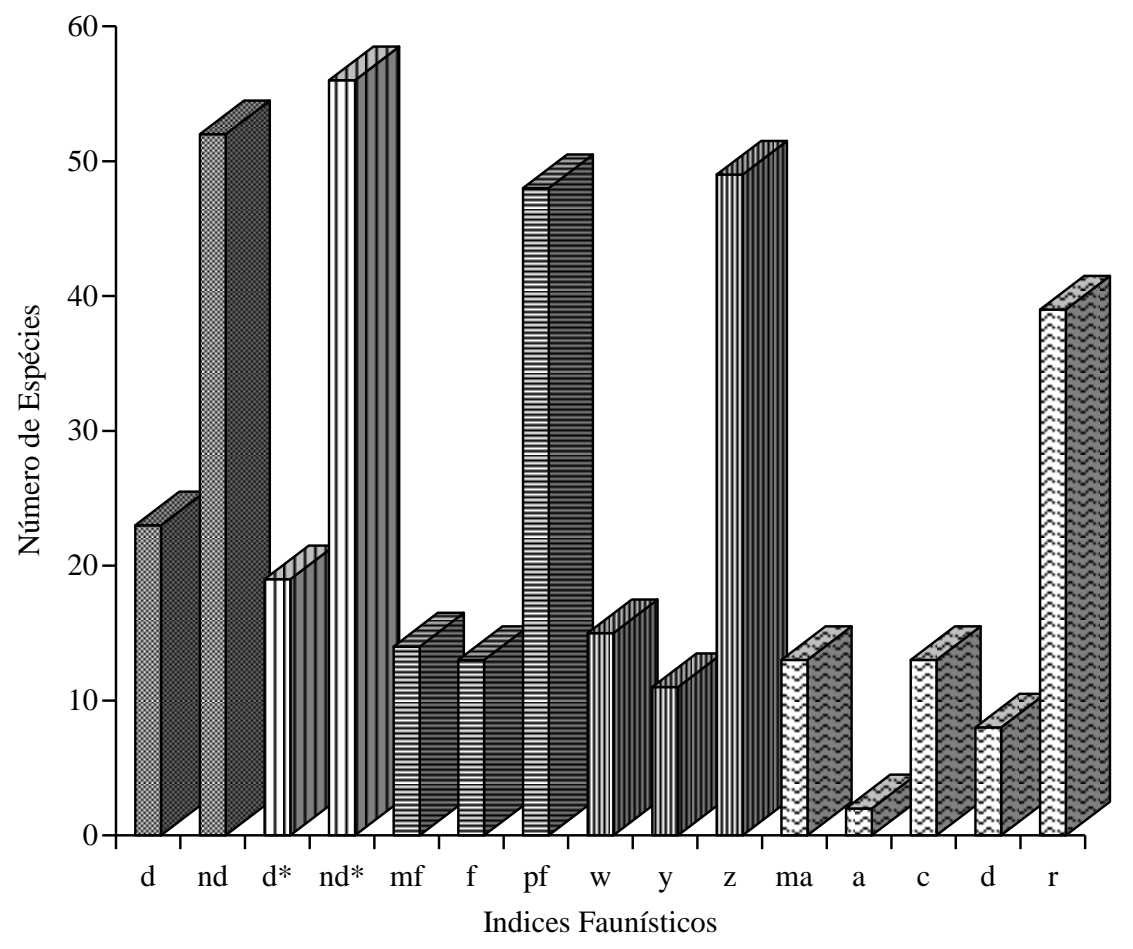

Figura 1. Quantidade de espécies das subfamílias de Nymphalidae dominantes, constantes, muito abundantes e muito freqüentes amostradas na Estação Ecológica de Iquê, Juína-MT, 2008 . 
Entre as espécies analisadas, Catonephele acontius, Catonephele numilia, Eunica sp. 1, Nica flavilla, Temenis laothe (Biblidinae), Archaeoprepona demophon (Chaxinae), Morpho achilles (Morphoinae), Fosterinaria sp., Harjesia sp., Harjesia obscura, Hermeuptychia hermes, Pseudodebis valentina, Taygetis tripunctata e Taygetis virgilia (Satyrinae) foram as mais importantes, pois ocorreram na análise faunística como muito frequente, constante e muito abundante (Tabela 1). Existe uma relação positiva entre a diversidade de espécies de ninfalídeos e a riqueza de espécies arbóreas na área de estudo, sugerindo que, independentemente do ambiente, a riqueza de borboletas frugívoras pode atuar como indicadora da biodiversidade. Segundo Raimundo et al. (2003), algumas espécies de borboletas frugívoras apresentam ótimas características como biondicadoras do estado de conservação em florestas primárias e espécies como $C$. acontius, a mais representativa neste levantamento, constitui ótima bioindicadora do estado de conservação em florestas primárias.

A espécie C. acontius foi, quantitativamente, a mais expressiva em número de indivíduos amostrados $\mathrm{e}$ apresentaram diferença pelo teste de Tukey a $5 \%$ de probabilidade em comparação às demais espécies. Harjesia sp. constituiu-se na segunda média mais representativa $\mathrm{e}$ diferenciou-se estatisticamente das quatro espécies testadas (Tabela 4).

O valor de 3,47 para o índice de Shannon-Wiener é considerado alto, indicando uma relação positiva entre a diversidade de espécies de ninfalídeos e a riqueza de espécies arbóreas na área, sugerindo que, independentemente do ambiente, a riqueza de borboletas frugívoras pode atuar como indicadora da biodiversidade (Tabela 5). A equitatividade de 0,80 mostra que a sazonalidade na oferta e na riqueza dos recursos alimentares, favoreceu as espécies mais generalistas e que, nesta comunidade, os indivíduos encontram-se melhor distribuídos nas populações, indicando uma provável ausência de dominância entre as espécies e uma distribuição mais homogênea dos recursos ambientais (Tabela 5). O mosaico vegetacional encontrado nesta região ecotonal forma uma grande variedade de hábitats, influenciando na distribuição das espécies e refletindo também na diversidade destas. O valor obtido para o índice de Shannon-Wiener $\left(\mathrm{H}^{\prime}\right)$ caracteriza, segundo Magurran (1988), um ambiente bastante diversificado na sua composição, com diversidade, riqueza e abundância de espécies de borboletas frugívoras.

Tabela 4. Médias populacionais das espécies de ninfalídeos mais representativos na Estação Ecológica de Iquê, Juína-MT, 2008.

\begin{tabular}{|c|c|c|}
\hline Subfamília & Espécie & Médias de Indivíduos* \\
\hline Biblidinae & Catonephele acontius & $19,8 \mathrm{a}$ \\
\hline Satyrinae & Fosterinaria sp. & $8,0 \mathrm{~b}$ \\
\hline Satyrinae & Harjesia sp. & $4,3 \mathrm{c}$ \\
\hline Biblidinae & Temenis laothe & $3,8 \mathrm{c}$ \\
\hline Satyrinae & Harjesia obscura & $3,6 \mathrm{c}$ \\
\hline Biblidinae & Eunica sp. & $3,5 \mathrm{c}$ \\
\hline Satyrinae & Taygetis tripunctata & $3,3 \mathrm{c}$ \\
\hline
\end{tabular}


Tabela 5. Índice de diversidade de acordo com a série de números de Hill e equitatividade das espécies de subfamílias de Nymphalidae amostradas com armadilhas Van SomerenRydon na Estação Ecológica de Iquê, Juína-MT, 2008.

\begin{tabular}{c|c|c|c|c|c}
\hline \multicolumn{7}{c}{ ÍNDICE DE DIVERSIDADE } \\
\hline N0 & $\lambda$ & $\mathrm{H}^{\prime}$ & $\mathrm{N} 1$ & $\mathrm{~N} 2$ & $\mathrm{E}$ \\
\hline 75 & 6,14 & 3,47 & 32,16 & 16,26 & 0,80 \\
\hline
\end{tabular}

Número de espécies (N0); Índice de Simpson ( $\lambda$ ); Índice de Shannon (H'); Número de espécies abundantes (N1); Número de espécies muito abundantes (N2); Equitatividade (E).

\section{CONCLUSÃO}

$\mathrm{O}$ alto índice de diversidade e a equitatividade da fauna de borboletas frugívoras encontrados associados à abundância de indivíduos e espécies de Satyrinae, importantes bioindicadores de paisagens, indicam ótimo estado de conservação da Estação Ecológica de Iquê, constituindo-se em refúgio da lepidopterofauna da Amazônia Matogrossense.

\section{AGRADECIMENTO}

Ao Instituto Brasileiro do Meio Ambiente (IBAMA/MT), pela permissão concedida para a realização deste trabalho dentro de uma unidade de conservação de uso indireto.

\section{REFERÊNCIAS BIBLIOGRÁFICAS}

BROWN JR., K. S. 1991. Conservation of neotropical environments: insects as indicators. In: COLLINS, N. M.; THOMAS, J. A. (Eds.).The conservation of insects and their habitats Londres: Academic Press, p. 349-404.

BROWN JR., K. S. 1997. Diversity, disturbance, and sustainable use of Neotropical forests: insects as indicators for conservation monitoring. Journal of Insect Conservation, v. 1, p. 25-42.

BROWN JR., K. S.; FREITAS, A. V. L. 2000a. Diversidade de Lepidoptera em Santa Teresa, Espírito Santo. Boletim do
Museu de Biologia Mello Leitão. Espírito Santo, v.11/12, p. 71-118

DEVRIES, P. J. 1987. The butterflies of Costa Rica and their natural history: Papilionidae, Pieridae, and Nymphalidae. New Jersey: Princeton Univ. Press. 327 p.

DEVRIES P. J., WALLA, T. 2001. Species diversity and community structure in neotropical fruit-feeding butterflies. Biological Journal of the Linnean Society, v.74, p. 1-15.

DOWNES, J. A. 1973. Lepidoptera feeding at puddle margins, dung and carrion. Journal of the Lepidopterist's Society, v.27, p. 89-99.

ELTON, C. S. 1973. The structure of invertebrate populations inside neotropical rainforest. Journal of Animal Ecology v.42. p.55-104.

FREITAS, A. V. L.; FRANCINI, R.; BROWN JR, K. S. 2003. Insetos como indicadores ambientais. In: CULLENJR., L.; RUDRAN, R.; VALLADARESPADUA, C. (Org.). Biologia da conservação \& Manejo da vida Silvestre. Curitiba: Editora da UFPR. Fundação $O$ Boticário de Proteção à Natureza. p. 125-148.

GILBERT, L. E; SINGER, M. C. 1975. Butterfly ecology. Annual Review Ecology System, v.6. p. 365-397.

LANDAU, B.; PROWELL, D.; CARLTON, C. 1999. Intensive versus long-term sampling to assess Lepidoptera diversity in southern mixed mesophytic forest. 
Annals of the Entomological Society of America, v.92, n.3. p. 435-441.

LAROCA, S; O. H. H. MIELKE. 1975. Ensaios sobre ecologia de comunidades em Sphingidae da Serra do Mar, Paraná, Brasil (Lepidoptera). Revista Brasileira de Biologia, 35(1):1-9.

LUDWIG, J. C.; REYNOLDS, J. F. 1988. Statistical ecology: a primer on methods and computing. $337 \mathrm{p}$.

MAGURRAN, A. E. 1988. Ecological diversity and its measurement. New Jersey:Princeton University Press. 179 p.

MACARTHUR, R. H. 1972. Fluctuation on animal populations and a measure of community stability. Ecology, v.36. p. 533-536.

MORAES, R. C. B.; SILVEIRA NETO, S. 2003. Software para análise faunística. In: Simpósio de Controle Biológico, 8, São Paulo, Resumos, Piracicaba-SP. ESALQ. p. 95.

PURVIS, A.; HECTOR, A. 2000. Getting the measure of biodiversity. Nature, v.405. p. 212-219.

RAIMUNDO, R. L. G.; FREITAS, A. V. L.; COSTA, R. N. S.; OLIVEIRA, J. B. F.; LIMA, A. F.; MELO, A. B.; BROWN JR., K. S. 2003. Manual de Monitoramento Ambiental usando borboletas e libélulas - Reserva Extrativista Alto do Juruá. Série Pesquisa e Monitoramento Participativo em áreas de conservação gerenciada por populações tradicionais. Campinas-SP, v. $1,36 \mathrm{p}$.

RIBEIRO, D. B. A. 2006. Guilda de borboletas frugívoras em uma paisagem fragmentada no Alto Paraíba, São Paulo Campinas, SP: 78 p. 2006. Dissertação de Mestrado em Ecologia, Universidade Estadual de Campinas, Campinas, São Paulo.

SAKAGAMI, S.F.; LAROCA, S. 1971. Observations on the bionomics of some Neotropical Xylocopini bees, with some comparative biofaunistic notes
(Hymenoptera, Anthophoridae). J. Fac. Sci. Hokkaiado Uni. 6. (Zool.) v. 18, p. 57-127.

SANTOS, E. C. S.; MIELKE, O. H. H; CASAGRANDE, M. M. 2008. Inventários de borboletas no Brasil: estado da arte e modelo de áreas prioritárias para pesquisa com vistas à conservação. Natureza \& Conservação, v.6, n.2. p. 68-90.

SCHOEREDER, J. H.; SOBRINHO, T. G.; RIBAS, C. R.; CAMPOS, R. B. F. 2004. Colonization and extinction of ant communities in a fragmented landscape. Austral Ecology, v.29. p. 391-398.

VIEIRA, L. M. S. 2004. Paisagem da Reserva Biológica Estadual Mata Paludosa como um recurso de educação ambiental. Porto Alegre, 2004. 45 p. Trabalho de Graduação (Bacharel em Geografia) - Universidade Federal do Rio Grande do Sul. 